\title{
Unsupervised Learning Architecture for Classifying the Transient Noise of Interferometric Gravitational- Wave Detectors
}

\section{Yusuke Sakai}

Tokyo City University

Yousuke Itoh

Osaka City University

\section{Piljong Jung}

National Institute for Mathematical Sciences

\section{Keiko Kokeyama}

Cardiff University

\section{Chihiro Kozakai}

National Astronomical Observatory of Japan

\section{Katsuko T. Nakahira}

Nagaoka University of Technology

\section{Shoichi Oshino}

Institute for Cosmic Ray Research, The University of Tokyo

Yutaka Shikano

Gunma University

Hirotaka Takahashi ( $\square$ hirotaka@tcu.ac.jp )

Tokyo City University

\section{Takashi Uchiyama}

Institute for Cosmic Ray Research, The University of Tokyo

Gen Ueshima

Nagaoka University of Technology

\section{Tatsuki Washimi}

National Astronomical Observatory of Japan

\section{Takahiro Yamamoto}

Institute for Cosmic Ray Research, The University of Tokyo

\section{Takaaki Yokozawa}

Institute for Cosmic Ray Research, The University of Tokyo 
Keywords: Unsupervised, Architecture, Transient Noise, Gravitational-wave

Posted Date: December 8th, 2021

DOI: https://doi.org/10.21203/rs.3.rs-1094374/v1

License: (c) (1) This work is licensed under a Creative Commons Attribution 4.0 International License. Read Full License 


\title{
Unsupervised Learning Architecture for Classifying the Transient Noise of Interferometric Gravitational-wave Detectors
}

\author{
Yusuke Sakai $^{1,+}$, Yousuke Itoh ${ }^{2,3}$, Piljong Jung ${ }^{4}$, Keiko Kokeyama $^{5}$, Chihiro Kozakai ${ }^{6}$, \\ Katsuko T. Nakahira ${ }^{7}$, Shoichi Oshino ${ }^{8}$, Yutaka Shikano ${ }^{9,10,11,}$ \\ Hirotaka Takahashi ${ }^{1}, 12,13,++$, Takashi Uchiyama ${ }^{8}$, Gen Ueshima ${ }^{7}$, Tatsuki Washimi ${ }^{6}$, \\ Takahiro Yamamoto ${ }^{8}$, and Takaaki Yokozawa ${ }^{8}$
}

\author{
${ }^{1}$ Research Center for Space Science, Advanced Research Laboratories, Tokyo City University, Setagaya-ku, \\ Tokyo 158-0082, Japan \\ ${ }^{2}$ Graduate School of Science, Osaka City University, Sumiyoshi-ku, Osaka City, Osaka 558-8585, Japan \\ ${ }^{3}$ Nambu Yoichiro Institute of Theoretical and Experimental Physics (NITEP), Osaka City University, Sumiyoshi-ku, \\ Osaka City, Osaka 558-8585, Japan \\ ${ }^{4}$ National Institute for Mathematical Sciences, Daejeon 34047, Republic of Korea \\ ${ }^{5}$ School of Physics and Astronomy, Cardiff University, The Parade, Cardiff, CF24 3AA United Kingdom \\ ${ }^{6}$ Gravitational Wave Science Project, Kamioka branch, National Astronomical Observatory of Japan, Hida City, \\ Gifu 506-1205, Japan \\ ${ }^{7}$ Department of Information and Management Systems Engineering, Nagaoka University of Technology, \\ Nagaoka, Niigata 940-2188, Japan \\ ${ }^{8}$ Institute for Cosmic Ray Research, KAGRA Observatory, The University of Tokyo, Hida City, Gifu 506-1205, \\ Japan \\ ${ }^{9}$ Graduate School of Science and Technology, Gunma University, Maebashi, Gunma 371-8510, Japan \\ ${ }^{10}$ Institute for Quantum Studies, Chapman University, Orange, CA 92866, USA \\ ${ }^{11}$ JST PRESTO, Kawaguchi, Saitama 332-0012, Japan \\ ${ }^{12}$ Institute for Cosmic Ray Research, The University of Tokyo, Kashiwa City, Chiba 277-8582, Japan \\ ${ }^{13}$ Earthquake Research Institute, The University of Tokyo, Bunkyo-ku, Tokyo 113-0032, Japan \\ +g2191402@tcu.ac.jp \\ ++hirotaka@tcu.ac.jp
}

\begin{abstract}
In the data of laser interferometric gravitational wave detectors, transient noise with non-stationary and non-Gaussian features occurs at a high rate. It often causes problems such as instability of the detector, hiding and/or imitating gravitational-wave signals. This transient noise has various characteristics in the time-frequency representation, which is considered to be associated with environmental and instrumental origins. Classification of transient noise can offer one of the clues for exploring its origin and improving the performance of the detector. One approach for the classification of these noises is supervised learning. However, generally, supervised learning requires annotation of the training data, and there are issues with ensuring objectivity in the classification and its corresponding new classes. On the contrary, unsupervised learning can reduce the annotation work for the training data and ensuring objectivity in the classification and its corresponding new classes. In this study, we propose an architecture for the classification of transient noise by using unsupervised learning, which combines a variational autoencoder and invariant information clustering. To evaluate the effectiveness of the proposed architecture, we used the dataset (time-frequency two-dimensional spectrogram images and labels) of the LIGO first observation run prepared by the Gravity Spy project. We obtain the consistency between the label annotated by Gravity Spy project and the class provided by our proposed unsupervised learning architecture and provide the potential for the existence of the unrevealed classes.
\end{abstract}

Gravitational waves are distortions of the spacetime propagating (most probably) at the speed of light. They are emitted from, e.g., coalescence of compact star binaries and supernova explosions. The first observation of a gravitational wave, which is from coalescence of a black hole binary, was achieved by the Laser Interferometer Gravitational-wave Observatory (LIGO) located in Livingston, Louisiana and Hanford, Washington in USA in September $2015^{2}$. Subsequently, LIGO and Virgo ${ }^{3}$ in 
Europe made three international joint observation runs and observed as many as 90 events of gravitational waves emitted by the coalescence of the compact binaries ${ }^{4,5,6,7}$. Moreover, GEO600 ${ }^{8}$ in Germany and KAGRA ${ }^{9}$ in Japan made a two-week observation run $(\mathrm{O} 3 \mathrm{GK})$ in April 2020. The subsequent fourth observation run (O4) is planned to be conducted jointly with LIGO, Virgo, and KAGRA.

When searching for a gravitational wave signal in the data from the interferometers, techniques of separating the gravitational waves from instrumental noise in the observed data are essential since the signals of the gravitational waves are generally smaller than the detector noise. The gravitational-wave detector is sensitive to environmental and instrumental states (such as ground motions, air pressure, optics suspensions, fluctuations in the laser, vacuum, mirror, etc.), and as a result, non-stationary and non-Gaussian noise, called "transient noise", frequently appears in the detector. Transient noise causes instability in the detector, hiding and/or imitating the gravitational-wave signals. The LIGO and Virgo collaboration reported that the transient noise with signal-to-noise ratio $>6.5$ occurred at the rate of 1.10 events per minute at LIGO Livingston (LLO) in the first half of third observation run (O3a) between 1 April 2019, 15:00 UTC and 1 October 2019, 15:00 UTC ${ }^{5}$, and 1.17 events per minute at LLO in the second half of O3 (O3b) between 1 November 2019, 15:00 UTC and 27 March 2020, 17:00 UTC ${ }^{7}$, respectively.

Transient noise has various time-frequency characteristics that are related to its causes in the detector. Classifying transient noise could provide us with one of the clues to explore its origins and to improve the performance of the detector. Among others, the Gravity Spy project ${ }^{10,11,12,13}$ is one of such efforts to classify transient noise. The Gravity Spy project used the Omicron software ${ }^{14}$ to identify the signal of transient noise observed in the time-series data. Thereafter, Omega Scan ${ }^{15} \mathrm{created}$ a time-frequency spectrogram around the identified transient noise as a two-dimensional (2D) image. Based on a part of these created 2D images, using cloud resources in collaboration with LIGO detector characterisation experts and volunteer citizen scientists for the analysis, 22 types of labels associated with the characteristics or causes of transient noise were annotated. Both images and labels were recorded. Finally, they classified the transient noise in the remaining images by supervised learning using the pre-classified images and labels. The data annotation for machine learning requires such a great deal of work.

The previous studies ${ }^{16}$ which analysed the feature embedding learned from the Gravity Spy dataset ${ }^{11}$ observed a different

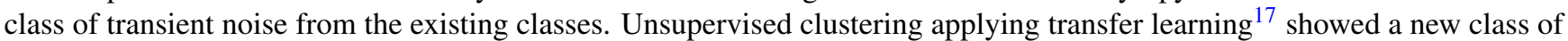
transient noise in addition to the 22 classes of the Gravity Spy project. Moreover, the supervised classification using the latest observation $\mathrm{O} 3$ dataset presented a new class of transient noise ${ }^{12}$.

Since unsupervised learning does not need any pre-assigned labels for the training dataset, this architecture is expected to reduce annotation work for the training data, to increase the objectivity of the classification and to even classify a new class such as the transient noise. In this study, we focus on unsupervised learning using a deep convolutional neural network and propose a classification architecture for transient noise. Our proposed architecture consists of two processes: feature learning and classification. In the feature learning process, the features of transient noise are extracted from the time-frequency spectrogram images (2D images) using a variational autoencoder (VAE) ${ }^{18,19}$. Regarding the classification process, invariant information clustering (IIC) ${ }^{20}$ is used to classify images of the transient noise using features extracted by the encoder of the pre-learned VAE. We apply the proposed architecture to the dataset ${ }^{11}$ created by the Gravity Spy project of the LIGO observation run $1(\mathrm{O} 1)^{4}$ as our input images, demonstrate the validity of the unsupervised classification result, and discuss the correspondence with the labels of the Gravity Spy project.

\section{Results}

The result section consists of two subsections: the results of the training process and evaluation of the unsupervised learning architecture. The Gravity Spy dataset of LIGO O1, which is presented by the Gravity Spy project shown in Figure 1, is used for training in our proposed architecture. This dataset contains a total of 8535 transient noises in four-time durations of 0.5 , 1.0, 2.0, and 4.0s. Each data has a label with one of the 22 types which are related to the origins or characteristics of the transient noise. The labels annotated by the Gravity Spy project under the Zooniverse, which is the online citizen science platform, are used only when evaluating the training results of the proposed architecture. In addition, the pre-processing of the dataset is shown in Section "Pre-process".

\section{Training Process of our Architecture}

We investigate the training parameters of the VAE to be determined as follows. The dimensions of the feature variable $z$ are $64,128,256,512$, and 1024; the training size rate is in the range of $[0.6,0.9]$ in increments of 0.1 ; the training rate is in the range of $\left[5 \times 10^{-7}, 5 \times 10^{-2}\right]$ in increments of one digit; the minibatch size is in the range of $[32,128]$ in increments of 32 . The maximisation of the lower bound Equation (3) (i.e. let $\delta=-\sum_{i}^{N} \mathscr{L}\left(\boldsymbol{x}^{(i)}, \boldsymbol{\theta}, \boldsymbol{\phi}\right)$ ) is used as a training objective, and the minimisation of $\delta$ is used for training. The value of $\delta$ does not have a significant effect on the dimension of $z$ and the training size rate. In contrast, the training rate and minibatch size are related to the value of $\delta$ and its stability. The representative 
parameters for training are shown at the left side of Figure 2 (a), and the training curves using these parameters are shown in Figure 2 (b). Considering the case 1 (black line in Figure 2 (b)), the training rate seems too low and $\delta$ does not decrease. Regarding the case 2 (grey line), the result of the training is not stable, showing the fluctuation in the curve, although $\delta$ has decreased compared with the Case 1 . In the case 3 (blue line), $\delta$ decreases in both the training and evaluation and seems stable after 100 epochs. Judging from these results above, for the rest of the study, the parameters of Case 3 are applied to the proposed architecture.

Examples of the reconstructed images of the transient noise generated by the decoder of the VAE at 100 epochs are shown in Figure 2 (c). The characteristics of the reconstructed images seem similar to those of the input images. We confirm a similar tendency for all the other inputs and reconstructed images. Therefore, the encoder of the VAE at 100 epochs is applied to the IIC for the classification of the transient noise.

Furthermore, the validity of the features by VAE is shown in Supplemental Material "Feature Visualization of Transient Noise using t-SNE" by visualised features $z$ which are projected using t-SNE.

After training the VAE, the training parameters of IIC are also investigated using the pre-trained encoder. The output classes are in the range of $[22,100]$ in increments of 2 ; the output over the classes is in range of $[50,500]$ in increments of 50; the classifier number is one of $3,5,10,20$; the training rate is in the range of $\left[5 \times 10^{-7}, 5 \times 10^{-2}\right]$ in increments of one digit; the minibatch size is in the range of $[64,256]$ in increments of 32 . Owing to the training, mutual information Equation (4) is convex with respect to the number of the output classes, which attains the maximum value when the output class is 36 . When the output over classes and the classifiers change, the mutual information does not seem to change. In this study, the IIC parameters shown at the left side of Figure 2 (a) (the maximum of the mutual information for the training) are used for the classification. In addition, considering the parameters of spectral clustering with multiple classifiers, the number of classifiers $K=5$, and the number of classes $C=36$. These are values when the mutual information is maximum. The training for the VAE and IIC with 128 mini-batch size takes approximately $1.0 \mathrm{~h} / 100$ epochs and approximately $0.3 \mathrm{~h} / 100 \mathrm{epochs,}$ respectively, using the two NVIDIA GeForce RTX 2080 Ti GPUs, Intel Xeon CPU E5-2637 v4 (core 8) and 125 GB of main memory.

\section{Evaluation of our Architecture}

The evaluation results are presented in this section. The proposed architecture shown in Section "Proposed Architecture" was trained using the pre-processing dataset described in Section "Pre-process".

Figure 3 shows a randomly selected image from each class (representative image) and similar images that have a high degree of similarity to the representative image in a class. These similar images are derived from the cosine similarity ${ }^{21}$ between the representative image and the other images, using an affinity matrix which is calculated by spectral clustering.

The representative images seem to have different characteristics for each class, and similar images are close to their representative images. Moreover, the image of class (15) in Figure 3 shows that the classifier recognises the same class even if the data are shifted in the time direction. Therefore, training that does not depend on the perturbation in the time duration is achieved using pre-processing the dataset.

To investigate the correspondence between the results of supervised and unsupervised learning, a confusion matrix using the Gravity Spy labels is shown in Figure 4. Considering the classes (classes (6) ("1080Lines"), (8) ("Repeating_Blips"), (14) ("Chirp"), (18) ("Helix"), and (24) ("Scratchy")), unsupervised learning classifies the Gravity Spy labels (noted parentheses) as one class. In addition, the output images by the classifier shown in Figure 3 are similar to those of the Gravity Spy labels.

The "Scattered_Light" class is separated into classes (2), (3), (11), and (16) on the confusion matrix, respectively. These classes are classified into different classes on unsupervised learning, whereas their characteristics are similar to Figure 3. A previous study ${ }^{12}$ on supervised learning with the Gravity Spy labels indicated the existence of a subclass that might be in the "Scattered_Light" class. The unsupervised classification yielded the same results as in the previous study, indicating the existence of a subclass of the "Scattered_Light" class.

Considering the "Blip" and "Koi_Fish" classes, both classes are separated into multiple classes as shown in Figure 4. The representative images and their similarity images from separating the classes are shown in Figure 5, where the similarity images are sorted in a descending order and are sampled randomly from the cosine similarity to the representative image. Each separating class is grouped into its own class, even for images with low cosine similarity. The images of the classes separated from "Blip" have a common Gravity Spy label. Moreover, the frequency growth of the spectrogram image for classes (9), (20), and (30) looks roughly similar, and the unsupervised classification classifies each class using their characteristics details. Similar results can be observed in "Koi_Fish" (class(5) and class(7)). Therefore, the images of "Blip" or "Koi_Fish" may be classified into more detailed subclasses.

The "Paired_Doves", "Wandering_Line", and "Air_Compressor" classes are few samples in the dataset (Figure 1 (b)). "Air_Compressor" is classified into one class; however, the other classes are not classified into any unique classes in the unsupervised classification. We assume that "Air_Compressor" is a class that cannot be divided anymore. Therefore, it is 
classified into one class, even with few data. On the contrary, "Paired_Doves" and "Wandering_Line" are assumed to have more subclasses. The reason why they are not classified into a specific class could be explained by the fact that there is a limited number of transient noises classified into "Paired_Doves" and "Wandering_Line".

The "None_of_the_Above" class of $\mathrm{O} 1$ dataset is a set of data that does not belong to any other Gravity Spy labels. The unsupervised classification does not classify these data into unique classes, and it distributes them into various kinds of classes. This result is consistent with the previous study ${ }^{11}$. In fact, the study ${ }^{12}$ using the $\mathrm{O} 3$ dataset $^{5}$ reports that a number of "None_of_the_Above" appears as "Blip" class or the new population of "Scattering_Light". A similar classification result is expected when applying our architecture to the $\mathrm{O} 3$ dataset and retraining it.

Based on the above results, the data of the Gravity Spy labels that are classified into multiple classes in unsupervised classification are shown as grey colour in the "Estimated number of class" in Figure 4. These data that are separated from the Gravity Spy labels may imply the existence of subclasses.

\section{Discussion}

Let the number of Gravity Spy classes (labels) be $C^{\prime}=22$, and let the classified result (vector) whose unsupervised class is the $i$-th class be $\boldsymbol{v}^{(i)} \in \mathbb{R}^{C^{\prime}}$, where $i \in c=\{0, \ldots, 35\}$. Alternatively, $v_{j}^{(i)}$, indicating the $j$-th component of $\boldsymbol{v}^{(i)}$, is the number of the $j$-th images, and the Gravity Spy label is classified as the $i$-th unsupervised class. The total number of classified $i$-th unsupervised classes is expressed by the $L^{1}$ norm ${ }^{21}$ of $\boldsymbol{v}^{(i)}$ (i.e., $\left|\boldsymbol{v}^{(i)}\right|_{1}=\sum_{j=1}^{C^{\prime}}\left|v_{j}^{(i)}\right|$ ). The $j$-th component of a normalised vector $\boldsymbol{v}^{(i)} /\left|\boldsymbol{v}^{(i)}\right|_{1}$ is the ratio of the $j$-th image of the Gravity Spy label on the $i$-th unsupervised class. Therefore, we define the accuracy of unsupervised learning as

$$
A=\sum_{i=1}^{C} \frac{\max \left(\boldsymbol{v}^{(i)}\right)}{\left|\boldsymbol{v}^{(i)}\right|_{1}}
$$

The accuracy of the proposed architecture was $90.9 \%$, where the total number of unsupervised classes was set to $C=36$. Comparatively, although Equation (1) is a slightly different definition from the usual definition of the accuracy of supervised learning, the supervised learning of the Gravity Spy project ${ }^{10}$ achieved $97.1 \%$ accuracy on the testing data using the same dataset used here.

We discuss the classification results in Figure 4, one of the factors that decrease the accuracy of unsupervised learning in Equation (1). The representative images of the major characteristics and images of their low similarities are shown in Figure 6. Considering classes (0) and (35), the classifier is able to identify the global features of images because the images are similar to the representative images that also exist in the data of other Gravity Spy labels. Regarding classes (13) and (34), the classifier cannot recognise the images properly and may be learning the background features. This problem can be solved by adjusting the neural-network configuration. Moreover, regarding class (26), it is observed that the minor images (such as "Power Line") are mixed with the major class ("Air Compressor"). The same result can also be observed for class (32). Because the characteristics of both images are similar, it is possible that both noises have similar characteristics. Additionally, a comparison of the classification results shown in Figure 4 with the feature visualisation using t-SNE is discussed in Supplemental Material "Feature Visualization of Transient Noise using t-SNE". Based on the above results, we confirm the consistency between the label annotated by Gravity spy project and the class provided by our proposed unsupervised learning architecture and provide the potential for the existence of the unrevealed classes.

Subsequently, we will build a system for the classification of transient noise using the proposed architecture in KAGRA. In addition, we will extend our architecture to self-supervised learning ${ }^{22}$ to enhance the accuracy of the classification. This algorithm trains the data of a specific label, known as the golden set ${ }^{10}$, which generates a pseudo label to the given dataset and retrains it. Using the new classes classified by unsupervised learning, the semi-supervised learning can help reduce the annotation process for the training and can solve the problem of ensuring objectivity in the classification. We would like to construct a semi-supervised architecture that would incorporate the advantages of both Gravity Spy's supervised and unsupervised learning.

\section{Method}

The proposed unsupervised learning method consists of two architectures: a variational autoencoder (VAE) and invariant information clustering (IIC). The VAE is used to learn the features from the time-frequency spectrogram (2D images) of transient noise, and the IIC classifies the transient noise from the features that are learned by the encoder of the VAE. Before we present the details of the method, we explain the target dataset. 


\section{Target dataset}

The Gravity Spy dataset ${ }^{11}$, which is the input data, is an image set of transient noise that is obtained from the LIGO O $1^{4}$. Omicron software ${ }^{14}$ searches for transient noise in time-series data, and Omega Scan ${ }^{15}$ software generates an image of the time-frequency spectrogram of each transient noise using Q-transformation ${ }^{15,23}$. Q-transformation is a method that estimates the frequency component of the time-series data by setting a window function on each time-frequency, generating a 2D image of the time-frequency spectrogram. The spectrogram image of each transient noise in the Gravity Spy dataset has four time durations $(0.5,1.0,2.0$, and $4.0 \mathrm{~s})$ at the centre as shown in Figure 1 (a). In addition, these transient noises are given 22 labels, which are related to cause as shown in Figure 1 (b). For example, the images of 12 classes of transient noise are shown in Figure 1 (c).

\section{Pre-process}

The pre-processing applied to the Gravity Spy dataset for training of our proposed architecture is shown in Figure 7.

1. For each transient noise, stack the images of the time-frequency spectrograms with the four-time widths as shown in Figure 7, and use it as the input data for this transient noise. The resolution of the transient noise image for each time duration is $224 \mathrm{px} \times 272 \mathrm{px}$ (frequency and temporal direction, respectively), and the dimensions of the stacked images are $4 \times 224 \times 272 \mathrm{px}$.

2. Convert the stacked data into two types:

Input Image : Crop the left and right parts of the image equally so that the resulting image has the dimension of 4 $\times 224 \mathrm{px} \times 224 \mathrm{px}$

Perturbed Image : Crop the left part of the image at the randomly time-shifted position of the range between 0-24px and also crop the right part of the image so that the resulting image has the dimension of $4 \times 224 \mathrm{px} \times 224 \mathrm{px}$

Considering the characteristics of the time-frequency spectrogram, a small displacement in the time direction does not change its physical characteristics because this operation can be interpreted as a change in the event time. Therefore, the time-shifted images can be regarded as new events of transient noise, and it makes the architecture realise the classification of transient noise that does not depend on small displacements in the time direction. On the contrary, a possible small displacement of the spectrogram in the frequency direction changes its physical characteristics. Therefore, the frequency-shifted images fall into different classes from the original one in the classification. Thus, the perturbation of transient noise is not applied in the frequency direction; nonetheless, they are applied only in the time direction.

In the training process of the proposed architecture, there is a random time shift of the image between 0-24 px used for the training data. The data that were cropped without a time shift were used for the evaluation of the VAE and the input image of the IIC.

\section{Variational Autoencoder}

In this study, the features of transient noise are obtained from their time-frequency 2D spectrogram image using VAE, one of the approaches for feature learning ${ }^{25,26}$ using convolutional deep learning. Generally, feature learning is a method for acquiring features that are effective for the prediction and classification of data. It also has the ability to convert highdimensional data to low-dimensional features.

Let the input dataset be $\mathscr{D}=\left\{\boldsymbol{x}^{(1)}, \ldots, \boldsymbol{x}^{(N)} \mid \boldsymbol{x}^{(i)} \in \mathbb{R}^{D}, i=1, \cdots, N\right\}$ and the marginal likelihood for $\mathscr{D}$ be $p_{\boldsymbol{\theta}}\left(\boldsymbol{x}^{(1)}, \ldots, \boldsymbol{x}^{(N)}\right)$, where $D$ is the dimension number, $N$ is the number of the input data, and $\theta$ are parameters for the architecture. The objective of the learning is to maximise the marginal likelihood. When the dataset $\mathscr{D}$ is independent and identically distributed, the $\log$ marginal likelihood becomes $\sum_{i=1}^{N} \ln p_{\boldsymbol{\theta}}\left(\boldsymbol{x}^{(i)}\right)$. Consider that the inference architecture $q_{\boldsymbol{\phi}}\left(\boldsymbol{z} \mid \boldsymbol{x}^{(i)}\right)$ (also known as encoder) approximates $q_{\boldsymbol{\phi}}\left(z \mid \boldsymbol{x}^{(i)}\right) \simeq p_{\boldsymbol{\theta}}\left(z \mid \boldsymbol{x}^{(i)}\right)$, where $z \in \mathbb{R}^{J}$ is a feature variable and $J<D$. Therefore, the log marginal likelihood $\ln p_{\boldsymbol{\theta}}\left(\boldsymbol{x}^{(i)}\right)$ can be expressed as

$$
\ln p_{\boldsymbol{\theta}}\left(\boldsymbol{x}^{(i)}\right)=\ln \int p_{\boldsymbol{\theta}}\left(\boldsymbol{x}^{(i)}, \boldsymbol{z}\right) d \boldsymbol{z} \geq \int q_{\boldsymbol{\phi}}\left(\boldsymbol{z} \mid \boldsymbol{x}^{(i)}\right) \ln \left(\frac{p_{\boldsymbol{\theta}}\left(\boldsymbol{x}^{(i)}, \boldsymbol{z}\right)}{q_{\boldsymbol{\phi}}\left(\boldsymbol{z} \mid \boldsymbol{x}^{(i)}\right)}\right) d \boldsymbol{z} \equiv \mathscr{L}\left(\boldsymbol{x}^{(i)}, \boldsymbol{\theta}, \boldsymbol{\phi}\right) .
$$

The second inequality is obtained by the Jensen's inequality, and $\mathscr{L}\left(\boldsymbol{x}^{(i)}, \boldsymbol{\theta}, \boldsymbol{\phi}\right)$ is an objective function known as the lower bound. Let a prior and a posterior distribution of $\boldsymbol{z}$ be a multivariate Gaussian distribution, indicating that $p_{\theta}(\boldsymbol{z})=\mathscr{N}(\boldsymbol{z} \mid \mathbf{0}, \boldsymbol{I})$ and $q_{\boldsymbol{\phi}}\left(\boldsymbol{z} \mid \boldsymbol{x}^{(i)}\right)=\mathscr{N}\left(\boldsymbol{z} \mid \boldsymbol{\mu}_{\boldsymbol{\phi}}\left(\boldsymbol{x}^{(i)}\right), \boldsymbol{\Sigma}_{\boldsymbol{\phi}}^{2}\left(\boldsymbol{x}^{(i)}\right) \boldsymbol{I}\right)$, where $\boldsymbol{\mu}_{\boldsymbol{\phi}}(\cdot)$ and $\boldsymbol{\Sigma}_{\boldsymbol{\phi}}(\cdot)$ are the outputs from an encoder and $\boldsymbol{I}$ is the identity matrix 
of dimension $J$. Let a posterior distribution of $\boldsymbol{x}$ be the multivariate Bernoulli distribution, $p_{\boldsymbol{\theta}}\left(\boldsymbol{x}^{(i)} \mid \boldsymbol{z}\right)=\operatorname{bern}\left(\boldsymbol{x}^{(i)} \mid \boldsymbol{g}_{\boldsymbol{\theta}}(\boldsymbol{z})\right)$, where $\boldsymbol{g}_{\boldsymbol{\theta}}(\cdot)$ are the outputs from the decoder. Thus, the expression of the lower bound to be maximised is

$$
\mathscr{L}\left(\boldsymbol{x}^{(i)}, \boldsymbol{\theta}, \boldsymbol{\phi}\right) \simeq-D_{\mathrm{KL}}\left(\mathscr{N}\left(\boldsymbol{z} \mid \boldsymbol{\mu}_{\boldsymbol{\phi}}\left(\boldsymbol{x}^{(i)}\right), \boldsymbol{\Sigma}_{\boldsymbol{\phi}}^{2}\left(\boldsymbol{x}^{(i)}\right) \boldsymbol{I}\right) \| \mathscr{N}(\boldsymbol{z} \mid \mathbf{0}, \boldsymbol{I})\right)+\frac{1}{L} \sum_{l=1}^{L} \ln \operatorname{bern}\left(\boldsymbol{x}^{(i)} \mid \boldsymbol{z}^{(i, l)}\right)
$$

where $D_{\mathrm{KL}}[\cdot \| \cdot \cdot]$ is the Kullback-Leibler divergence of two distributions and $z^{(i, l)}$ is referred to as the reparameterization trick, such that $\boldsymbol{z}^{(i, l)}=\boldsymbol{g}_{\boldsymbol{\phi}}\left(\boldsymbol{\varepsilon}^{(l)}, \boldsymbol{x}^{(i)}\right)=\boldsymbol{\mu}_{\boldsymbol{\phi}}\left(\boldsymbol{x}^{(i)}\right)+\boldsymbol{\varepsilon}^{(l)} \odot \boldsymbol{\Sigma}_{\boldsymbol{\phi}}\left(\boldsymbol{x}^{(i)}\right)$, where $\boldsymbol{\varepsilon} \sim \mathscr{N}(\mathbf{0}, \boldsymbol{I})$, and $\odot$ means Hadamard product.

\section{Classification using Invariant Information Clustering}

A typical method for clustering is the $k$-means which uses the Euclidean distances between data. Recently, a family of the $k$ means have been developed (e.g., $k$-means $++^{27}$, fuzzy $c$-means ${ }^{28}, x$-means ${ }^{29}$, etc.). Regarding clustering in a high dimensional space, the variance of the distance between data becomes small owing to the "curse of dimensionality". Alternatively, IIC ${ }^{20}$, which is a classification method, seems to be effective because it does not use distances of the data for learning. In this study, transient noise is classified using IIC by maximising the mutual information. Let $x \in \mathbb{R}^{D}$ be the input data, $\boldsymbol{x}^{\prime}$ be the perturbed data of $\boldsymbol{x}, C$ the number of output classes, and $\boldsymbol{\Phi}(\boldsymbol{x}) \in \mathbb{R}^{C}$ be a classifier in which the output layer of the classifier uses the SoftMax activation function. Consider a pair of cluster assignments for two inputs, $\boldsymbol{x}$ and $\boldsymbol{x}^{\prime}$. Their conditional joint distributions and marginal distributions are $P_{i j}=\boldsymbol{\Phi}\left(\boldsymbol{x}^{(i)}\right) \cdot \boldsymbol{\Phi}\left(\boldsymbol{x}^{(j) \prime}\right)^{\mathrm{T}}$ and $P_{i}=\sum_{j} \boldsymbol{\Phi}\left(\boldsymbol{x}^{(i)}\right) \cdot \boldsymbol{\Phi}\left(\boldsymbol{x}^{(j)^{\prime}}\right)^{\mathrm{T}}$, respectively, where the superscript $\mathrm{T}$ denotes the transpose. The objective for the maximisation of the mutual information is expressed as

$$
\max _{\boldsymbol{\Phi}} I\left(\boldsymbol{\Phi}(\boldsymbol{x}), \boldsymbol{\Phi}\left(\boldsymbol{x}^{\prime}\right)\right)=\sum_{i}^{C} \sum_{j}^{C} P_{i j} \ln \frac{P_{i j}}{P_{i} P_{j}}
$$

To improve the performance of the classifier, auxiliary over-clustering ${ }^{20}$ is also used when calculating the mutual information. This over-clustering formula is the same as Equation (4), except for $\boldsymbol{\Phi}(\boldsymbol{x}) \in \mathbb{R}^{W}$, where $C<W$.

\section{Proposed Architecture}

We propose an unsupervised classification as shown in Figure 8. It is a deep learning architecture that trains time-frequency 2D spectrogram images of transient noise. Considering the proposed architecture, the feature variables of the input image $\boldsymbol{x}$ and its perturbation image $\boldsymbol{x}^{\prime}=\boldsymbol{\xi}(\boldsymbol{x})$ are extracted by a pre-trained encoder of the VAE. The perturbation $\boldsymbol{\xi}$ is a transformation that does not change the information required for the classification (see Section "Pre-process"). Subsequently, the IIC learns to maximise the mutual information $I\left(\boldsymbol{\Phi}(\boldsymbol{z}), \boldsymbol{\Phi}\left(\boldsymbol{z}^{\prime}\right)\right)$, which is composed of a pair of feature variables $\left(\boldsymbol{z}=\boldsymbol{\mu}_{\boldsymbol{\phi}}(\boldsymbol{x}), \boldsymbol{z}^{\prime}=\boldsymbol{\mu}_{\boldsymbol{\phi}}\left(\boldsymbol{x}^{\prime}\right)\right)$.

The clustering of the IIC depends on the initial values of the neural networks in which the values are randomly provided. Thus, the classification results from each classifier varies slightly. Regarding the unsupervised learning, it is difficult to apply an ensemble average for each classification result to solve the dependencies of the initial values because the classified labels are random at each time. In this study, spectral clustering ${ }^{30}$ was applied to compress the multiple results of classification into one result. The approaches are:

1. Let $D, K$, and $C$ be the number of datasets, number of classifiers, and estimated number of classes, respectively. Create a hypermatrix $H$ whose dimension is $(D, K \times C)$ from each classifier result.

2. Considering $h^{(i)}$ as a row vector for each data of $H$, calculate an affinity matrix using the Gaussian kernel, which is given by $\exp \left(-\left\|h^{(i)}-h^{(j)}\right\|\right)$.

3. Compute the spectral clustering to the affinity matrix. Consequently, we have labels for each dataset whose dimension is $(D, C)$.

\section{References}

1. Aasi, J. et al. Advanced LIGO. Class. quantum gravity 32, 074001, DOI: 10.1088/0264-9381/32/7/074001 (2015).

2. Abbott, B. et al. GW150914: The Advanced LIGO detectors in the era of first discoveries. Phys. Rev. Lett. 116, 131103, DOI: 10.1103/PhysRevLett.116.131103 (2016).

3. Acernese, F. a. et al. Advanced Virgo: a second-generation interferometric gravitational wave detector. Class. Quantum Gravity 32, 024001, DOI: 10.1088/0264-9381/32/2/024001 (2014).

4. Abbott, B. et al. GWTC-1: A gravitational-wave transient catalog of compact binary mergers observed by LIGO and Virgo during the first and second observing runs. Phys. Rev. X 9, 031040, DOI: 10.1103/PhysRevX.9.031040 (2019). 
5. Abbott, R. et al. GWTC-2: Compact binary coalescences observed by LIGO and Virgo during the first half of the third observing run. Phys. Rev. X 11, 021053, DOI: 10.1103/PhysRevX.11.021053 (2021).

6. Abbott, R. et al. GWTC-2.1: Deep extended catalog of compact binary coalescences observed by LIGO and Virgo during the first half of the third observing run. arXiv preprint arXiv:2108.01045 (2021).

7. Abbott, R. et al. GWTC-3: Compact binary coalescences observed by LIGO and Virgo during the second part of the third observing run. arXiv preprint arXiv:2111.03606 (2021).

8. Grote, H., Collaboration, L. S. et al. The status of GEO 600. Class. Quantum Gravity 25, 114043, DOI: 10.1088/ 0264-9381/21/5/006 (2008).

9. Akutsu, T. et al. KAGRA: 2.5 generation interferometric gravitational wave detector. Nat. Astron. 3, 35, DOI: 10.1038/ s41550-018-0658-y (2019).

10. Zevin, M. et al. Gravity Spy: Integrating advanced LIGO detector characterization, machine learning, and citizen science. Class. Quantum Gravity 34, 064003, DOI: 10.1088/1361-6382/aa5cea (2017).

11. Bahaadini, S. et al. Machine learning for Gravity Spy: Glitch classification and dataset. Inf. Sci. 444, 172-186, DOI: 10.1016/j.ins.2018.02.068 (2018).

12. Soni, S. et al. Discovering features in gravitational-wave data through detector characterization, citizen science and machine learning. Class. Quantum Gravity 38, 195016, DOI: 10.1088/1361-6382/ac1ccb (2021).

13. Bahaadini, S. et al. Deep multi-view models for glitch classification. 2017 IEEE Int. Conf. on Acoust. Speech Signal Process. (ICASSP) 2931-2935, DOI: 10.1109/ICASSP.2017.7952693 (2017).

14. Robinet, F. et al. Omicron: A tool to characterize transient noise in gravitational-wave detectors. SoftwareX 12, 100620, DOI: 10.1016/j.softx.2020.100620 (2020).

15. Chatterji, S., Blackburn, L., Martin, G. \& Katsavounidis, E. Multiresolution techniques for the detection of gravitationalwave bursts. Class. Quantum Gravity 21, S1809, DOI: 10.1088/0264-9381/21/20/024 (2004).

16. Bahaadini, S. et al. Direct: Deep discriminative embedding for clustering of ligo data. 2018 25th IEEE Int. Conf. on Image Process. (ICIP) 748-752, DOI: 10.1109/ICIP.2018.8451708 (2018).

17. George, D., Shen, H. \& Huerta, E. Classification and unsupervised clustering of LIGO data with deep transfer learning. Phys. Rev. D 97, 101501, DOI: 10.1103/PhysRevD.97.101501 (2018).

18. Kingma, D. P. \& Welling, M. Auto-encoding variational bayes. arXiv preprint arXiv:1312.6114 (2013).

19. Kingma, D. P. \& Welling, M. An introduction to variational autoencoders. Foundations Trends Mach. Learn. 12, 307-392, DOI: $10.1561 / 2200000056(2019)$.

20. Ji, X., Henriques, J. F. \& Vedaldi, A. Invariant information clustering for unsupervised image classification and segmentation. Proc. IEEE Int. Conf. on Comput. Vis. 2019-October, 9865-9874, DOI: 10.1109/ICCV.2019.00996 (2019).

21. Murphy, K. P. Machine learning: a probabilistic perspective (MIT press, 2012).

22. Lee, D.-H. Pseudo-label: The simple and efficient semi-supervised learning method for deep neural networks. In Workshop on challenges in representation learning, ICML, vol. 3, 896 (2013).

23. Brown, J. C. Calculation of a constant Q spectral transform. J. Acoust. Soc. Am. 89, 425-435, DOI: 10.1121/1.400476 (1991).

24. Taylor, L. \& Nitschke, G. Improving deep learning with generic data augmentation. In 2018 IEEE Symposium Series on Computational Intelligence (SSCI), 1542-1547, DOI: 10.1109/SSCI.2018.8628742 (IEEE, 2018).

25. Zhong, G., Wang, L.-N., Ling, X. \& Dong, J. An overview on data representation learning: From traditional feature learning to recent deep learning. The J. Finance Data Sci. 2, 265-278, DOI: 10.1016/j.jfds.2017.05.001 (2016).

26. Bengio, Y., Courville, A. \& Vincent, P. Representation learning: A review and new perspectives. IEEE Transactions on Pattern Analysis Mach. Intell. 35, 1798-1828, DOI: 10.1109/TPAMI.2013.50 (2013).

27. Arthur, D. \& Vassilvitskii, S. $k$-means++: The advantages of careful seeding. Proc. Annu. ACM-SIAM Symp. on Discret. Algorithms 07-09-January-2007, 1027-1035 (2007).

28. Bezdek, J. C., Ehrlich, R. \& Full, W. Fcm: The fuzzy c-means clustering algorithm. Comput. Geosci. 10, 191-203, DOI: 10.1016/0098-3004(84)90020-7 (1984).

29. Radwan, A. et al. $x$-means clustering for wireless sensor networks. J. Robotics, Netw. Artif. Life 7, 111-115, DOI: 10.2991/jrnal.k.200528.008 (2020). 
30. Von Luxburg, U. A tutorial on spectral clustering. Stat. computing 17, 395-416, DOI: 10.1007/s11222-007-9033-Z (2007).

31. Kingma, D. P. \& Ba, J. Adam: A method for stochastic optimization. arXiv preprint arXiv:1412.6980 (2014).

\section{Acknowledgements}

We are grateful to the members of the Gravity Spy project for enlightening discussions. This study was supported in part by the Inter-University Research Program of Institute for Cosmic Ray Research, University of Tokyo, Japan. It was also supported in part by the Japan Society for the Promotion of Science (JSPS) Grants-in-Aid for Scientific Research on Innovative Areas, Grant No. 24103005 [JP17H06358, JP17H06361 and JP20H04731], by JSPS Core-to-Core Program A, Advanced Research Networks, and by JSPS KAKENHI [Grant No. 19H0190 (YI and HT) and Nos. 19K14636 and 21H05599 (Y. Shikano)], by JST, PRESTO [Grant No. JPMJPR20M4 (Y. Shikano) ].

\section{Author contributions}

Y Sakai, GU, and HT conceptualized the study; Y Sakai, GU, Y Sikano, TU, and HT framed the methodology; Y Sakai, GU, and HT concentrated on the software development and analysis; YI, PJ, KK, CK, KTN, SO, Y Shikano, TU, TW, T Yamamoto and T Yokozawa performed the validation and investigation; KK, CK, SO, TW, T Yamamoto and T Yokozawa made some critical and technical suggestions; Y Sakai, GU, and HT prepared the initial draft of the manuscript; all the authors, mainly Y Sakai, YI, Y Shikano, HT, and TU wrote, reviewed, and edited the manuscript; HT supervised the study; HT and TU administered the project. All authors have read and agreed to the published version of the manuscript.

\section{Competing interests}

The authors declare no competing interests.

\section{Data availability}

All the results are reported for public data of Gravity Spy project. Data on the results of unsupervised learning are available upon request from Y. Sakai and HT.

\section{Code availability}

All the codes developed in this study are available upon request to Y. Sakai and HT.

\section{Additional information}

Correspondence and requests for materials should be addressed to Y. Sakai (g2191402@tcu.ac.jp) and HT (hirotaka@tcu.ac.jp). Reprints and permissions information is available at www.nature.com/reprints.

Publisher's note Springer Nature remains neutral with regard to jurisdictional claims in the published maps and institutional affiliations. 
(a) Four time duration of a transient noise in Gravity Spy dataset

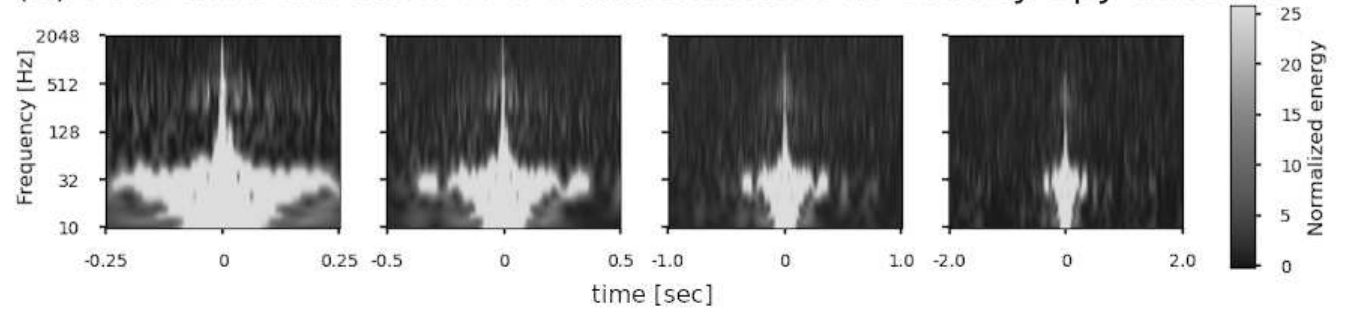

(b) Classes in the Gravity Spy dataset

\begin{tabular}{|lll|lll|}
\hline Class & Total & Rate & Class & Total & Rate \\
\hline \hline 1080Lines & 328 & $3.82 \%$ & No_Glitch & 181 & $2.11 \%$ \\
1400Ripples & 232 & $2.70 \%$ & None_of_the_Above & 88 & $1.03 \%$ \\
Air_Compressor & 58 & $0.68 \%$ & Paired_Doves & 27 & $0.31 \%$ \\
Blip & 1869 & $21.78 \%$ & Power_Line & 453 & $5.28 \%$ \\
Chirp & 66 & $0.77 \%$ & Repeating_Blips & 285 & $3.32 \%$ \\
Extremely_Loud & 454 & $5.29 \%$ & Scattered_Light & 459 & $5.35 \%$ \\
Helix & 279 & $3.25 \%$ & Scratchy & 354 & $4.12 \%$ \\
Koi_Fish & 830 & $9.67 \%$ & Tomte & 116 & $1.35 \%$ \\
Light_Modulation & 573 & $6.68 \%$ & Violin_Mode & 472 & $5.50 \%$ \\
Low_Frequency_Burst & 657 & $7.65 \%$ & Wandering_Line & 44 & $0.51 \%$ \\
Low_Frequency_Lines & 453 & $5.28 \%$ & Whistle & 305 & $3.55 \%$ \\
\hline
\end{tabular}

(c) Sample of each transient noise in the Gravity Spy dataset
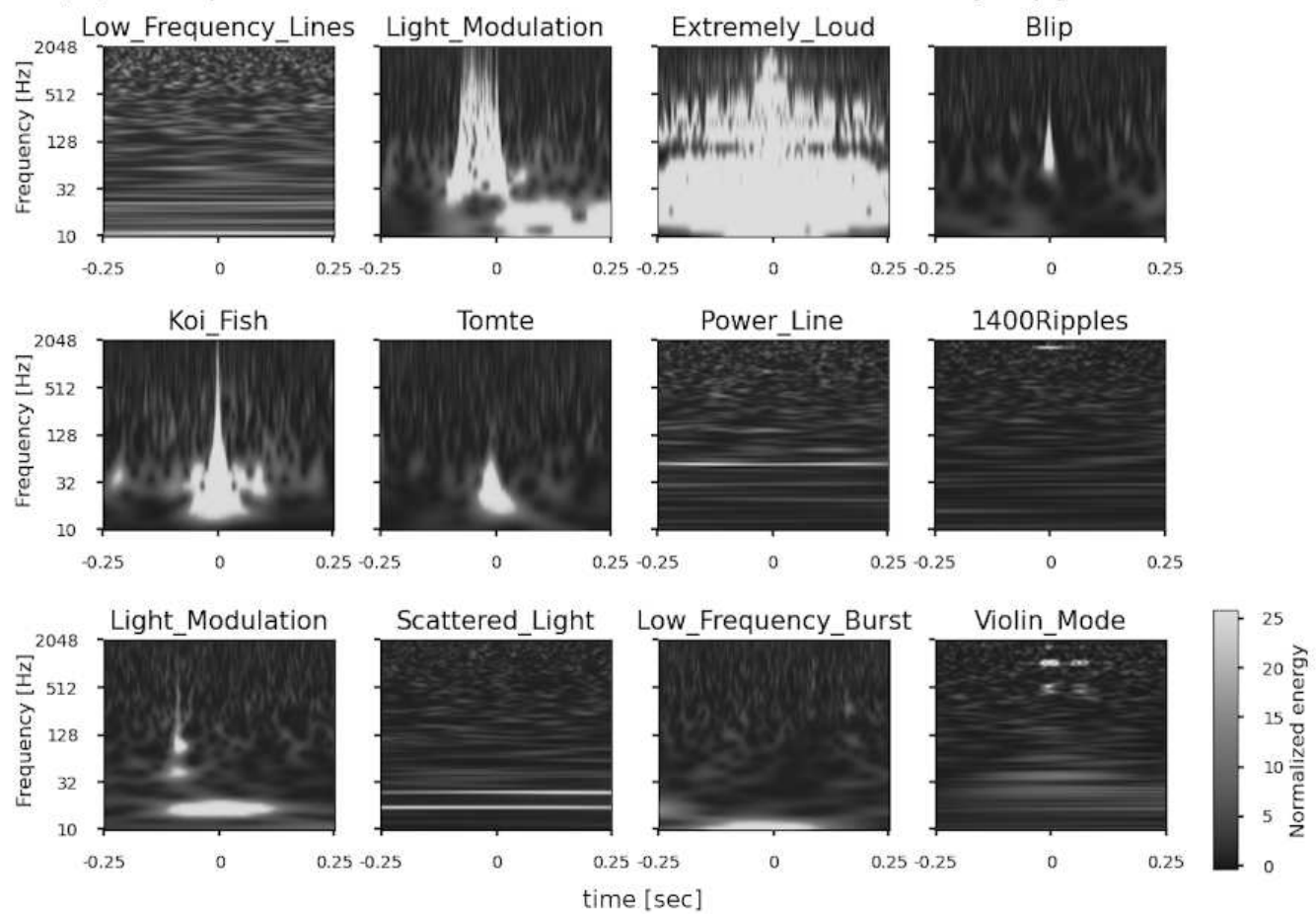

Figure 1. (a) Example of 2D image of the time-frequency spectrogram of transient noise in the Gravity Spy dataset. Regarding each transient noise, four time durations $(0.5,1.0,2.0$ and $4.0 \mathrm{~s}$ from the left of the figure) are recorded from the centre time. (b) Table showing all the classes, the number of data, and its ratio to the number in the Gravity Spy dataset.

There are 22 classes in total, and each of the 21 classes is given a name related to an occurrence cause or a characteristics of the shape on the spectrogram of transient noise. The other is "None_of_the_Above", which does not belong to any class. (c) Example of the image for each class in the Gravity Spy dataset. The figure shows 12 of the 22 classes of the transient noise with $0.5 \mathrm{~s}$. 
(a) Parameters for the proposed architecture

\begin{tabular}{llll}
\hline Parameters of VAE & case1 & case2 & case 3 \\
\hline Dimension of $z$ & 256 & 128 & 512 \\
Training size rate & 0.7 & 0.6 & 0.8 \\
Training rate of optimizer & $5 \times 10^{-7}$ & $5 \times 10^{-2}$ & $5 \times 10^{-4}$ \\
Minibatch size & 96 & 32 & 128 \\
\hline \hline
\end{tabular}

\begin{tabular}{ll}
\hline Parameters of IIC & \\
\hline \hline Output classes & 36 \\
Output over classes & 250 \\
Classifier number & 5 \\
Training rate of optimizer & $5 \times 10^{-4}$
\end{tabular}

Minibatch size

128

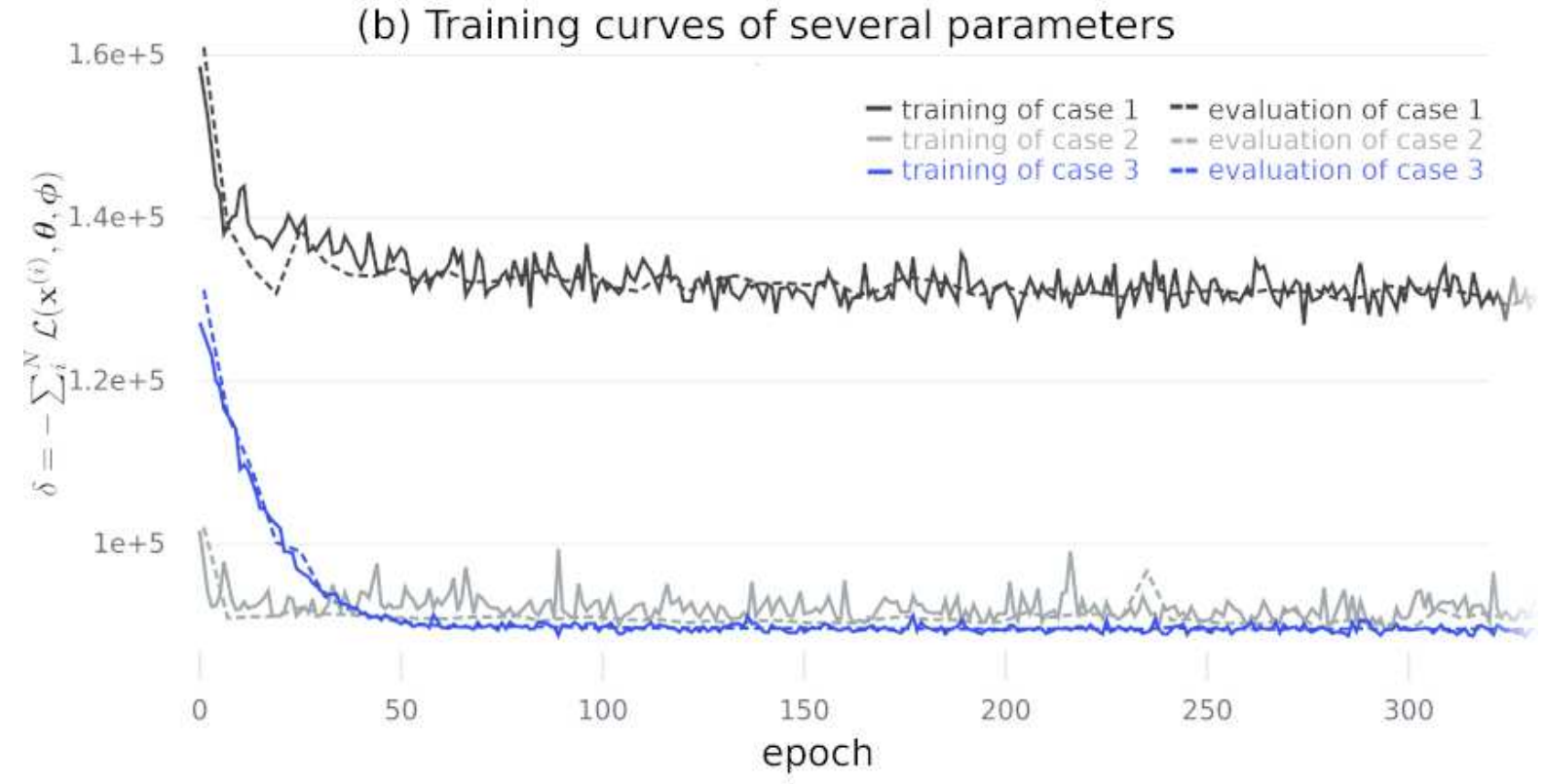

(c) Reconstruct images from the decoder of proposed architecture Sample of input image
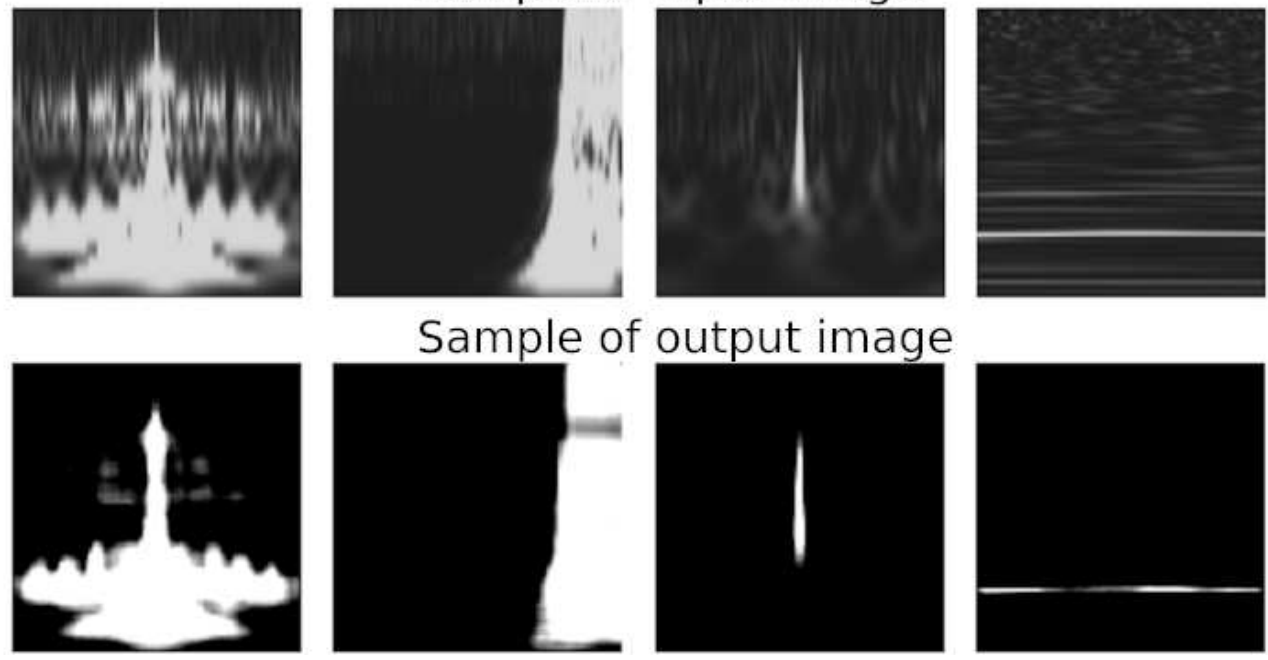

Figure 2. Left (a) Training parameters for the VAE of the proposed architecture. The dimension of $z$ is the output number of the encoder. The training size rate is the ratio of the total number of data to the data size of the input at training. Regarding the architecture evaluation, the input size is set to $(1-$ Training size rate). The training rate is the initial training rate, and the optimizer used is Adam ${ }^{31}$. Right (a) Training parameters for the IIC of the proposed architecture. The output classes is set to the number of classes to be classified. The classifier number is for multiple classifiers that are used to improve the performance of the classifier using spectral clustering. (b) Training curve during the training and evaluation of the VAE. The solid and dashed lines in the figure show the training objective $\delta \equiv-\sum_{i}^{N} \mathscr{L}\left(\boldsymbol{x}^{(i)}, \boldsymbol{\theta}, \boldsymbol{\phi}\right)$ at the time of training and evaluation, respectively. (c) Reconstructed image generated by the decoder of the VAE at 100 epochs in case 3. 


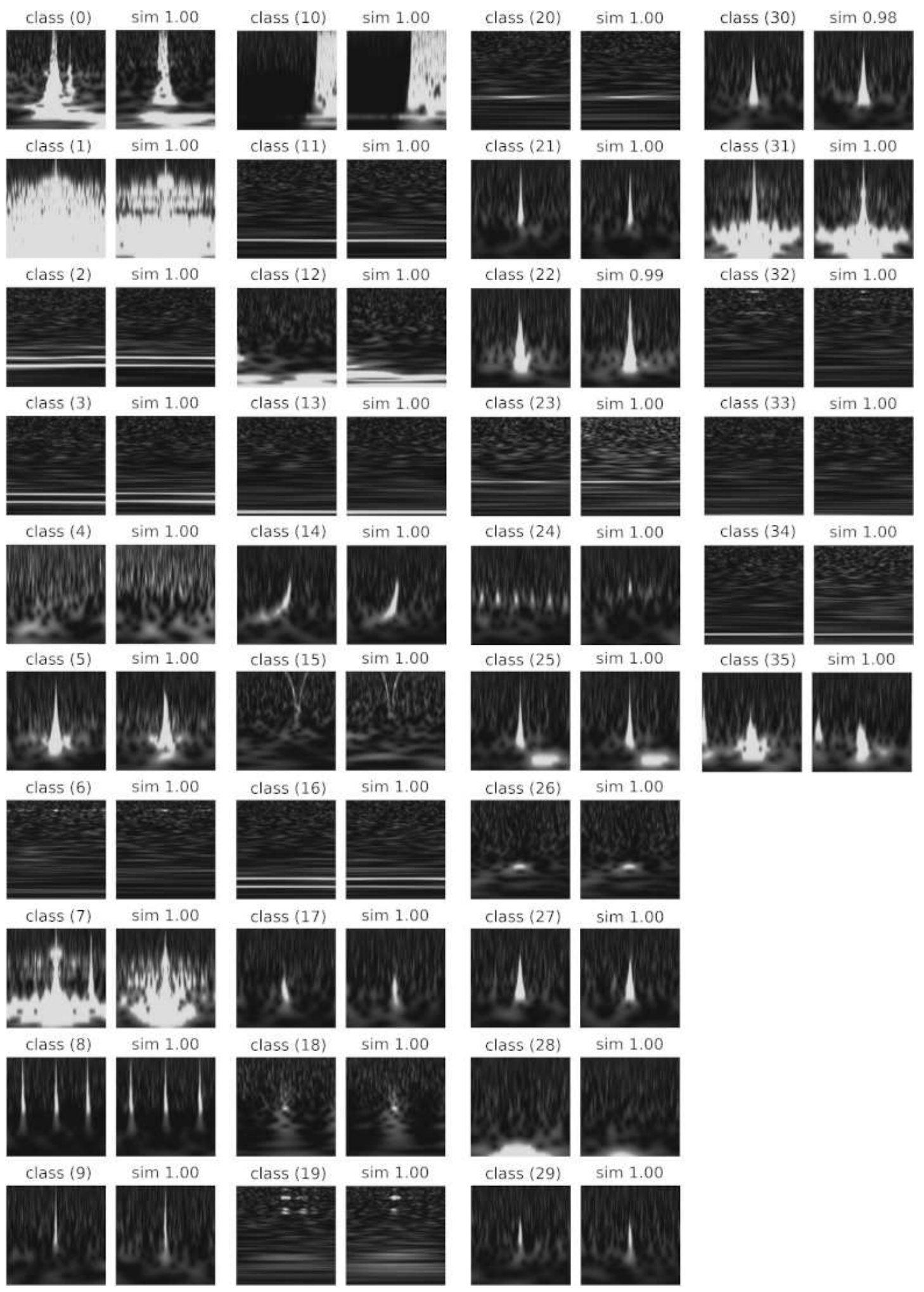

Figure 3. The representative and similar images in all the classes were classified using unsupervised learning. This representative image which is denoted by $i$ in the image is randomly selected from a class $i \in c=\{0, \ldots, 35\}$, and its most similar image is to the right of a representative image in class $(i)$. A cosine similarity to the representative image in class $i$ is shown at the top of the image. 
Number of data for each unsupervised label

Estimated

윙

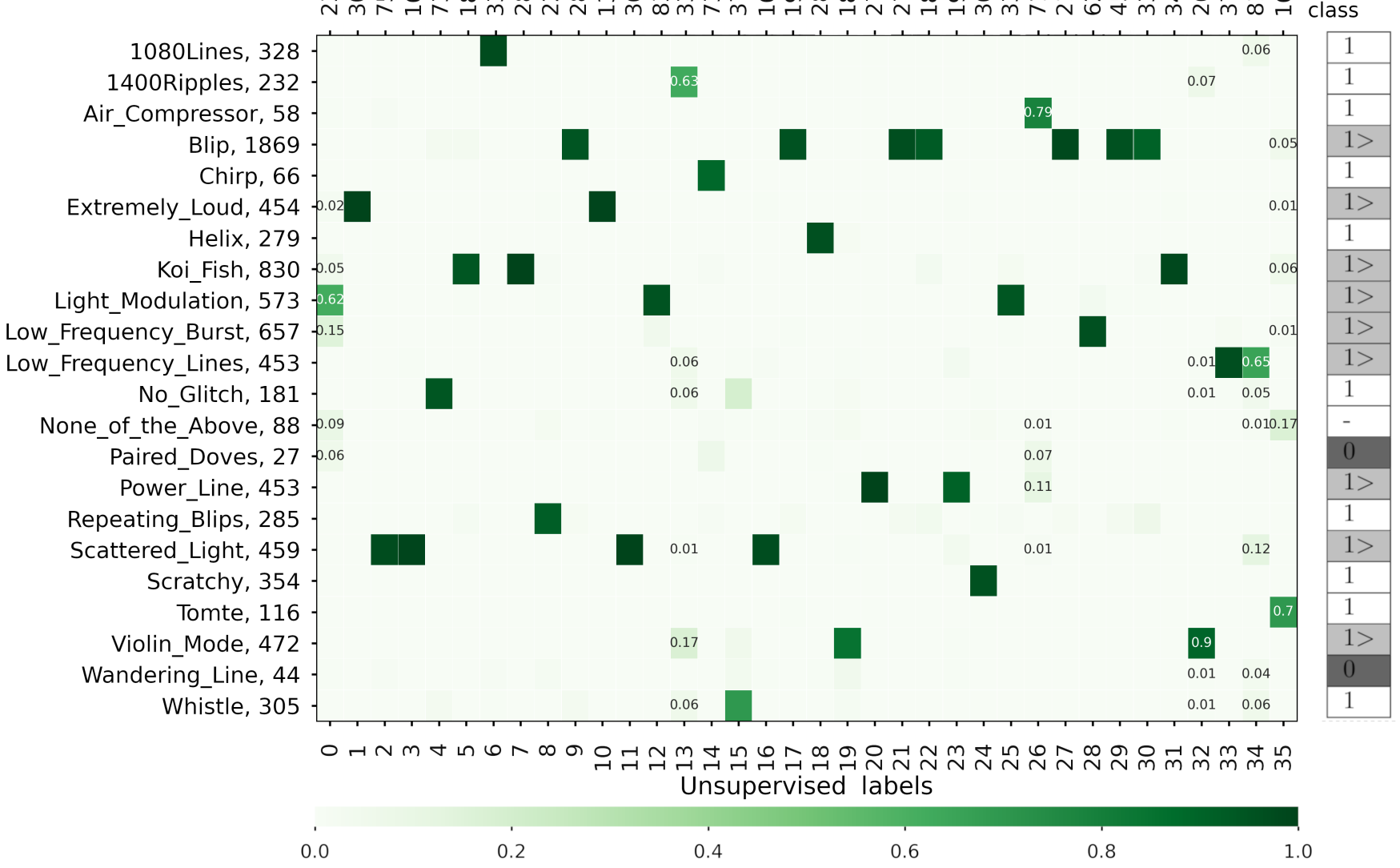

Figure 4. Confusion matrix of the classification results of the proposed architecture. The vertical axis of the confusion matrix represents the labels and number of data of the Gravity Spy dataset. The lower and upper horizontal axes denote the number of images classified into the unsupervised classes and the labels of the unsupervised classes, respectively. Each column of the confusion matrix is coloured using the ratio of the Gravity Spy-labelled images classified into the unsupervised class $(i)$. In addition, the classes that are separated from the Gravity Spy labels on the confusion matrix, such that classes (0), (13), (26), (32), (35), and (36) also show the ratio values in the matrix. The potential number of classes on Gravity Spy labels which are estimated by unsupervised learning are shown in the right column of the figure. The notation "1" (coloured on white) indicates that the number of classes labelled by the Gravity Spy matches the result of the unsupervised learning, and the inequality sign (coloured in light grey) indicates that the class is separated into multiple classes in the unsupervised learning. The notation " 0 " (coloured in dark grey) indicates an unclassified class in this training and dataset, and "-" notation indicates that they do not belong to any class of the unsupervised learning. 

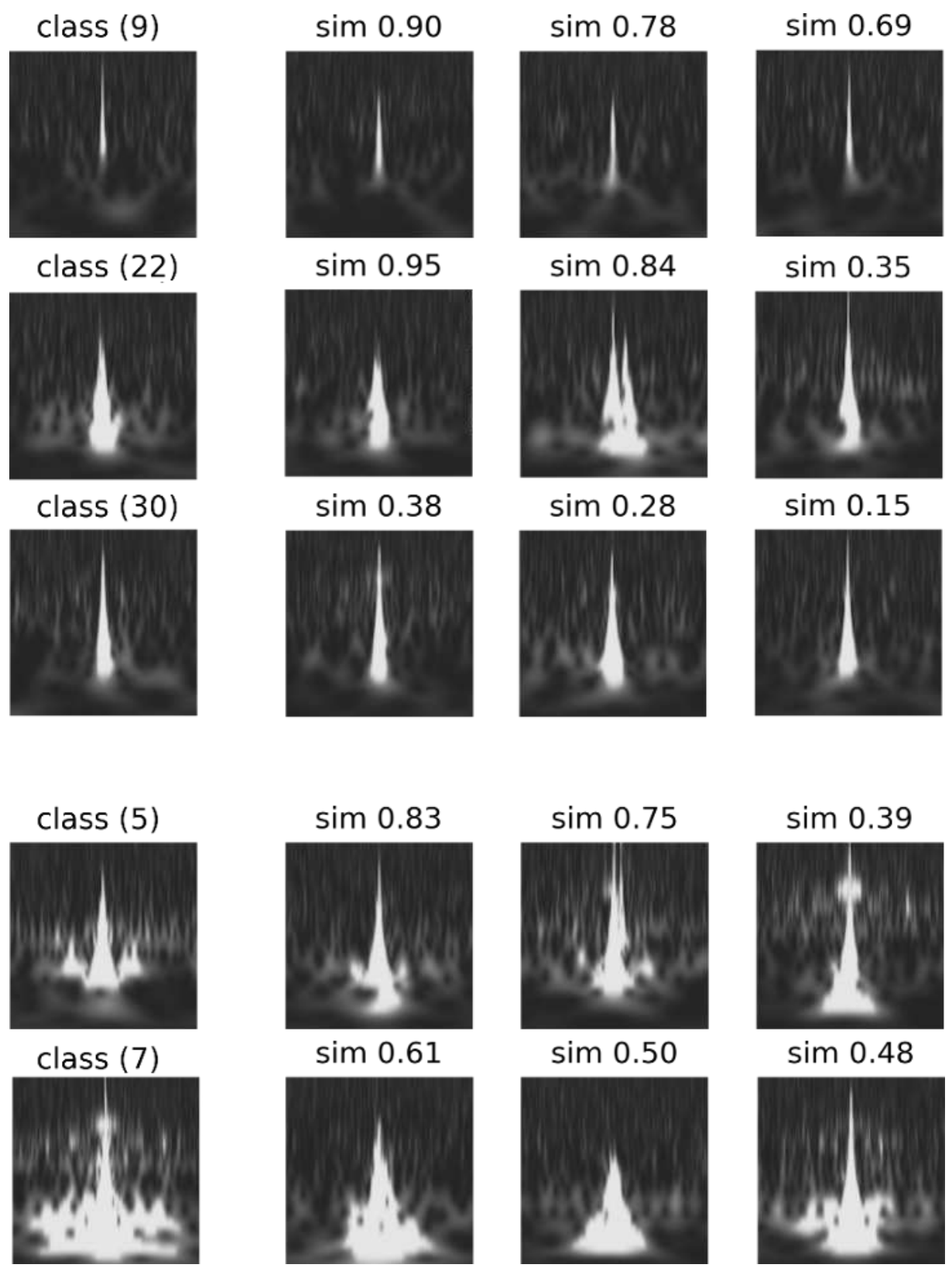

Figure 5. Representative images and images similar to unsupervised learning. Considering the figure, classes (9), (22), and (30) are separated from the "Blip" class, and classes (5) and (7) are separated from the"Koi_Fish" class. The representative images in the left column are sampled randomly from the images classified in class $(i)$ using unsupervised learning. The similar images in the other columns are sorted in a descending order and are sampled randomly from the cosine similarity (a value at the top of an image), considering the representative image. 


\section{(a) class (0) and class (35)}

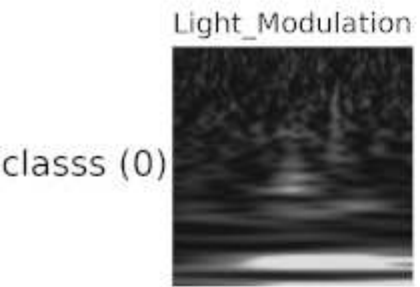

Tomte

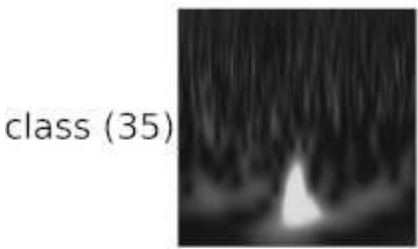

1400Ripples

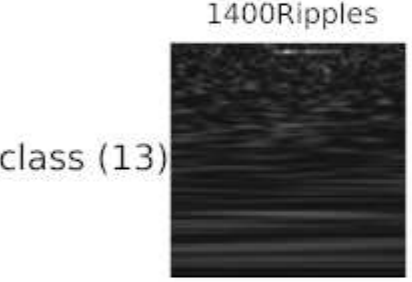

Low_Frequency_Lines

class $(34)$

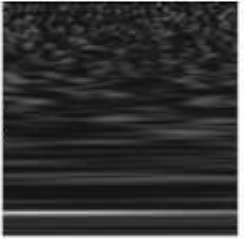

Air Compressor

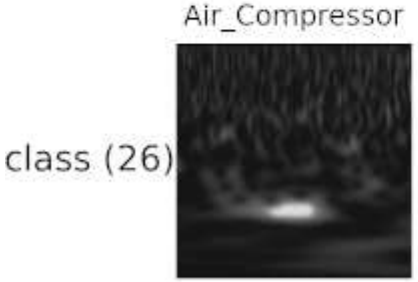

Violin Mode



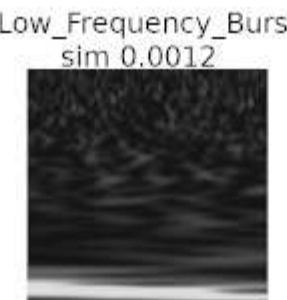

None of the Above $\operatorname{sim} 0.4040$

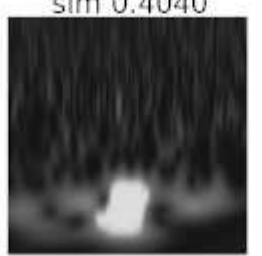

(b) class (13) and class (34)

Low_Frequency_Lines Low_Frequency_Lines $\operatorname{sim} 0.0136$

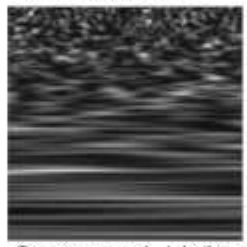

Scattered_Light $\operatorname{sim} 0.0 \overline{1} 91$

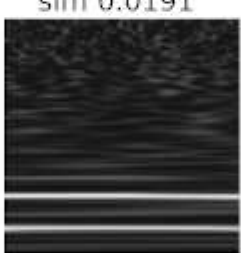

(c) class (26) and class (32)

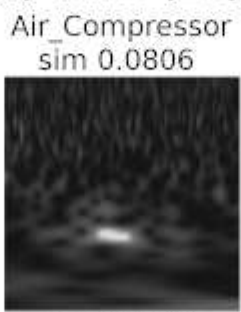

1400Ripples $\operatorname{sim} 0.6020$

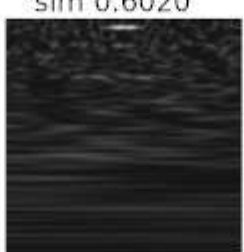

sim 0.0136

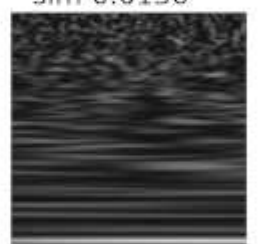

Wandering_Line $\operatorname{sim} 0.01 \overline{8} 8$



Power Line $\operatorname{sim} 0.0475$

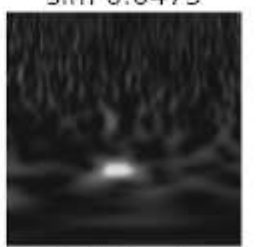

1400Ripples $\operatorname{sim} 0.3770$

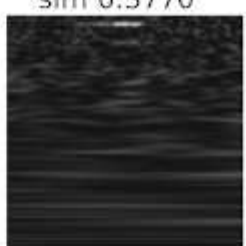

Low_Frequency_Burst

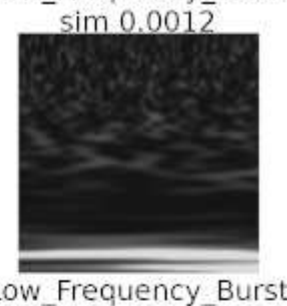

Low Frequency

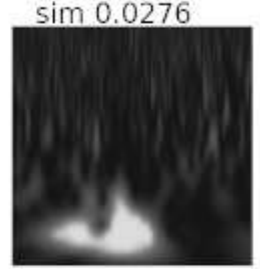

No_Glitch $\operatorname{sim} 0.0130$

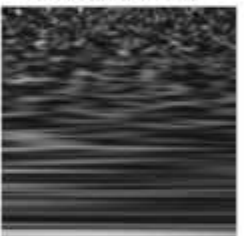

Wandering_Line $\operatorname{sim} 0.01 \overline{8} 4$

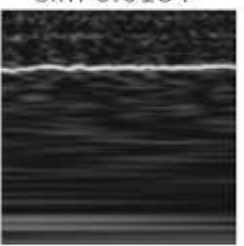

Power Line $\operatorname{sim} 0 . \overline{0} 475$

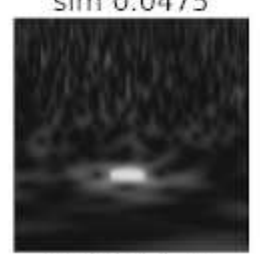

Violin Mode $\operatorname{sim} 0.0927$

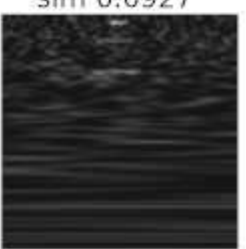

Figure 6. Examples of images in the classes with reduced accuracy in unsupervised learning. The major images in the left column are randomly sampled data from class $i$. The minor images in the other columns are sorted in an ascending order from the cosine similarity to its major image, indicating that they are sampled from the lowest similarity to the major one. The Gravity Spy label and the value of the cosine similarity are on top of the sampled image. 


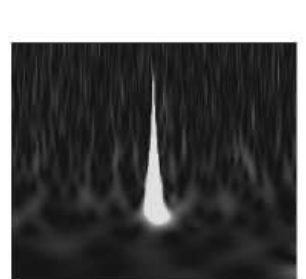

$0.5 \mathrm{sec}$

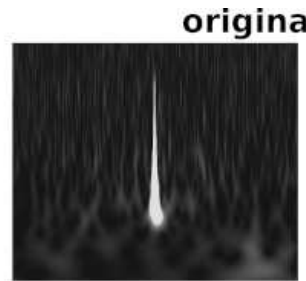

$1.0 \mathrm{sec}$

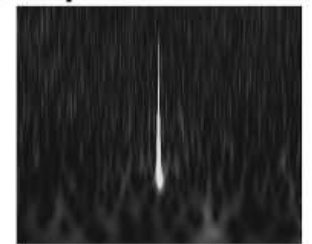

$2.0 \mathrm{sec}$

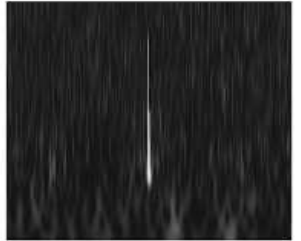

$4.0 \mathrm{sec}$

Stack samples

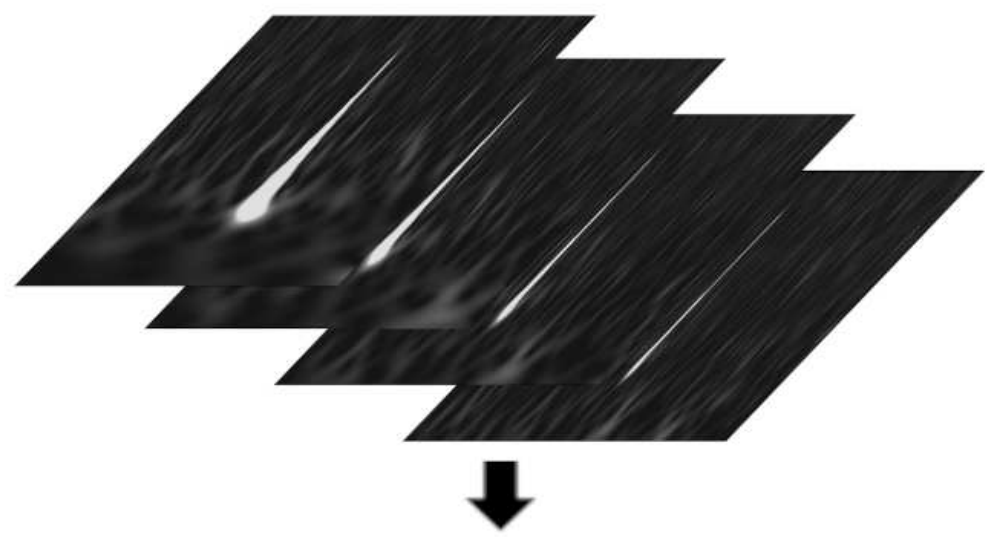

272px

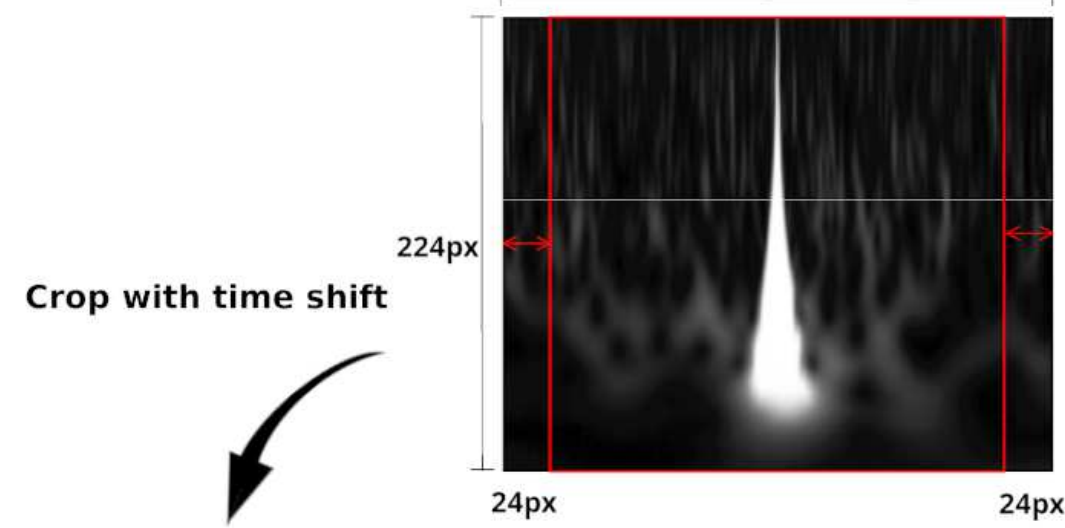

Samples for training

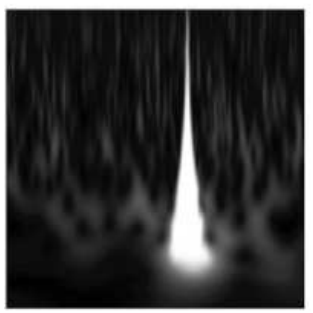

$-24 p x$



$+24 p x$

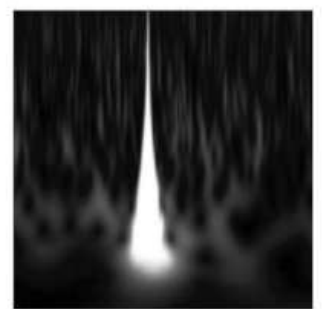

$+12 \mathrm{px}$
Crop without time shift

\section{Crop without time shift}

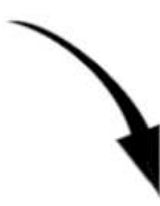

\section{Sample for evaluation}

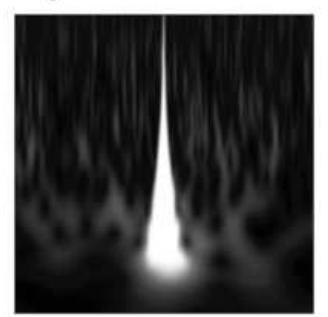

Figure 7. Overview of the input data pre-processing. The original samples are stacked in four time durations $(0.5,1.0,2.0$, and $4.0 \mathrm{~s}$ ) to generate the data, where the vertical and height of each image is $224 \mathrm{px}$ and $272 \mathrm{px}$, respectively, and the dimensions of the stacked data are 4, 224, and 272. Considering the training process of the proposed architecture, after a random time shift of image between 0-24 px, the dimensions of the data $(4,224$, and 224) are cropped and are used for the training data. The data that are cropped without a time shift are used for the evaluation of the VAE and as the input image of the IIC. 


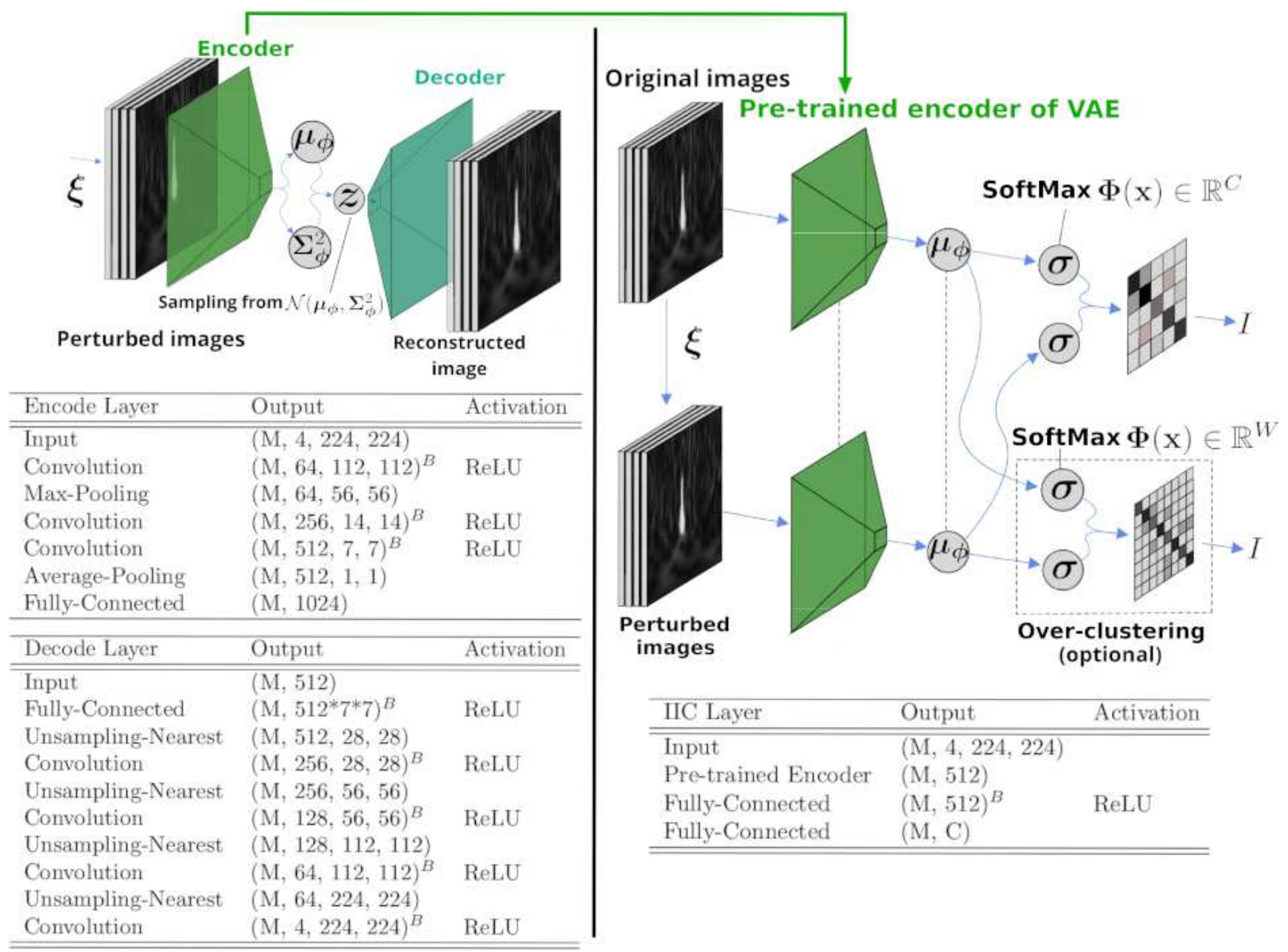

Figure 8. Proposed architecture for the classification of the transient noise. The tables show the details of the architectures of neural networks. ${ }^{B}$ denotes batch-normalize to an object, and $M$ denotes the mini-batch size. Left: Schematic architecture of the VAE for feature learning. The VAE trains neural networks to maximise the lower bound in Equation (3). The input to the VAE is a perturbed image $\boldsymbol{x}^{\prime}$ of the time-frequency spectrogram of the transient noise. This pre-process allows the encoder to learn features that do not depend on the perturbation. At the output layer of the encoder, the average and variance of the feature variable $z$ are output from the same network and separated into the dimensions (M, 512). Subsequently, the feature variables $z$ are constructed using the reparameterization trick. The decoder uses $z$ to generate a reconstructed image that is close to the input image. Right: Schematic architecture of the IIC for classification. The IIC trains neural networks to maximise the mutual information between the input data and its perturbed data. The inputs to the pre-trained encoders of the VAE are the original and perturbed images, respectively. Both encoders have the same architecture, and the dashed lines indicate the sharing weights of the neural networks in the figure. The IIC classifies transient noise using the SoftMax activation function at the output layer from the feature, which is the output of the pre-trained encoder. $C$ is the estimated number of classes of the transient noise, and $W$ is the number of classes used in the over-clustering. 


\section{Supplementary Files}

This is a list of supplementary files associated with this preprint. Click to download.

- 20211201SM.pdf 